\title{
Optimisation de l'utilisation du polyéthylène glycol, marqueur du flux dans l'intestin du ruminant
}

\author{
M Halbouche, J Brun-Bellut
}

Laboratoire science animale INRA-ENSAIA, 2 Avenue de la Forêt de Haye, 54500 Vandoeuvre, France

Le dosage du Polyéthylène Glycol (PEG), marqueur du flux intestinal, serait source de sous estimation de ce flux selon Smith (1958, Nature, 182, 260-261) à cause d'une interférence avec des substances du contenu digestif. Le principe turbidimétrique de son dosage est instable, et nécessite une optimisation.

Six échantillons de contenus du duodénum, marqués au PEG-4000 (30 g/j, 21 jours), provenant d'un bélier Texel et d'une chèvre Alpine chamoisée fistulés (rumen, duodénum) ont été traités pour le dosage de PEG selon Hyden (1956, Kungl Lantbruk shögskolans Annaler, 22, 139-145) et selon Smith (1958). La ration contenait des pulpes de betteraves déshydratées, de l'orge et du foin (50: 25: 25). Pour tester la réponse en densité optique (DO), 6 séries de 7 gammes étalon (GE) chacune $(0,100,200,300$ et 400 PPM) ont été constituées et traitées selon Hyden. Les GE de chaque série ont été lues sur turbidimètre après $10,20,30,45,60,90$ et $120 \mathrm{~min}$ de formation de turbidité.
Les 2 méthodes de dosage testées ont fourni des concentrations en PEG dans le contenu du duodénum similaires. La méthode Smith $a$, au contraire, provoqué une plus grande dispersion des répétitions. Le maximum de linéarité des GE est atteint entre 30 et $60 \mathrm{~min}(r=0,999)$, mais les pentes et les origines des régressions sont modifiées en raison de vitesses de développement de la turbidité différentes. La lecture devrait s'effectuer entre 45 et $60 \mathrm{~min}$ (pente maximale, origine proche de 0 ). Dans les GE, le seuil de turbidité maximum est d'autant plus instable et plus rapidement atteint que la concentration en PEG est forte (30 min pour 400 PPM, 60 min pour 100 PPM) ; ceci explique l'évolution des régressions et impose de ramener les concentrations en PEG des prises d'essai du contenu intestinal à des seuils inférieurs à 200 PPM.

En conclusion, les 2 méthodes testées ont été équivalentes. II est préconisé de traiter des prises d'essai contenant moins de 200 PPM de $P E G$, et de standardiser les délais d'attente avant lecture.

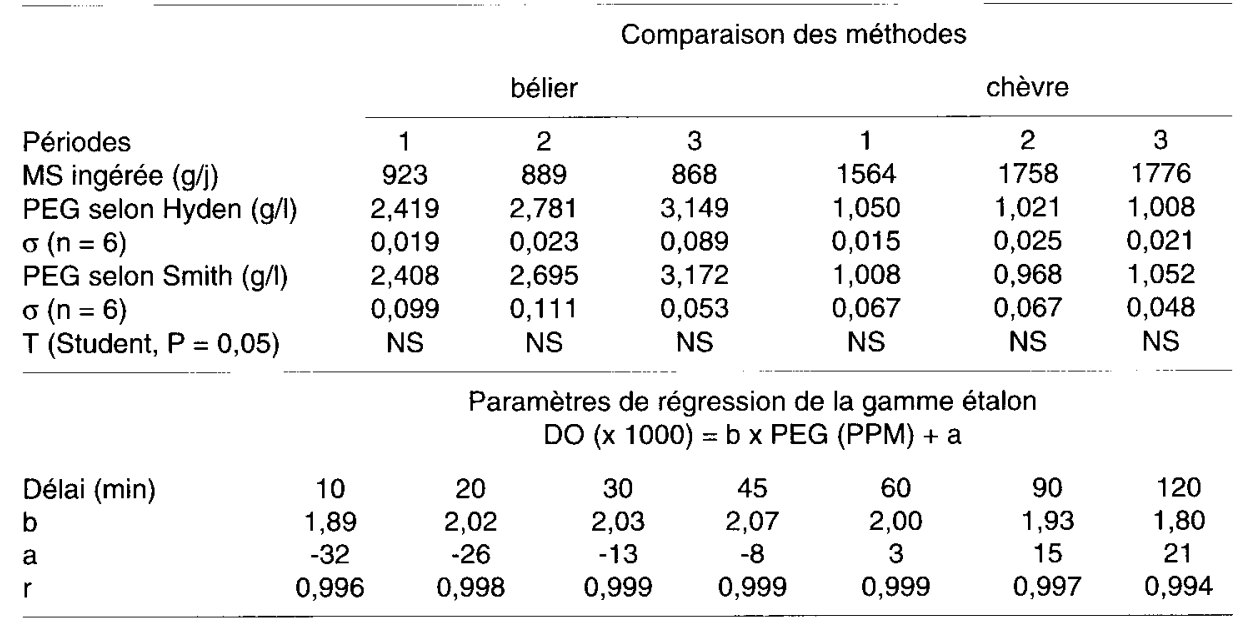

